\title{
Numerical Computation of Manipulator Singularities
}

\author{
Oriol Bohigas, Dimiter Zlatanov, Lluís Ros, Montserrat Manubens and Josep M. Porta
}

\begin{abstract}
This paper provides a method to compute all types of singularities of non-redundant manipulators with non-helical lower pairs and designated instantaneous input and output speeds. A system of equations describing each singularity type is given. Using a numerical method based on linear relaxations, the configurations in each type are computed independently. The method is general and complete: it can be applied to manipulators with arbitrary geometry; and will isolate singularities with the desired accuracy. As an example, the entire singularity set and its complete classification are computed for a two-degree-of-freedom mechanism. The complex partition of the configuration space by various singularities is illustrated by three-dimensional projections.

Index Terms-Singularity set computation, non-redundant manipulator, linear relaxation, branch-and-prune method.
\end{abstract}

\section{INTRODUCTION}

In robot singularities either the forward or the inverse instantaneous kinematic problem becomes undeterminate; the properties of the mechanism changing dramatically, and often detrimentally. The rich literature on singularity analysis, a central topic in Robot Kinematics, does not provide a general algorithm to explicitly compute and classify the singularity set of any given mechanism. Most works, including previous methods for computing singularities, focus on particular classes of singularities, and on specific robot architectures [1]-[13].

In [14], a general singularity classification was attempted, based on an input-output velocity equation. This overlooks cases where the motion of the mechanism cannot be described only with the input and output speeds. In $[15,16]$ more general methods from differential geometry were applied, and three singularity types were proposed. In [17, 18], a general manipulator model, in terms of differentiable mappings between manifolds, allowed a rigorous mathematical definition of kinematic singularity. Three basic types of singularities are identified: configuration-space, input, and output singularities, but the approach also allows a finer classification using six types, corresponding to various important physical and kinematic phenomena that may occur in a singularity. Although various conditions for the presence of singularities of all types have been presented [18, 19], no practical method for singularity computation has been proposed. The present work is aimed at completing this task.

O. Bohigas, L. Ros, M. Manubens and Josep M. Porta are with the Kinematics and Robot Design Group at the Institut de Robòtica i Informàtica Industrial, CSIC-UPC, Llorens Artigas 4-6, 08028 Barcelona, Spain. Emails: \{obohigas,lros,mmanuben,porta\}@iri.upc.edu.

D. Zlatanov is with the PMAR Laboratory (DIMEC) at the Università di Genova, Via Opera Pia 15a, 16145 Genoa, Italy.

This work has been partially supported by the Spanish Ministry of Economy through the contract DPI2010-18449, and by a Juan de la Cierva contract supporting the fourth author.
The rest of the paper is organized as follows. Section II provides some necessary background for the paper, in order to derive, in Section III, a system of equations characterizing each singularity type. Section IV describes a numerical method based on linear relaxations able to isolate the singular configurations encoded by each system obtained. The performance of this method is then illustrated in Section V on a particular manipulator. Section VI, finally, provides the main conclusions of the paper.

\section{BACKGROUND}

Every manipulator configuration can be described by a vector $\boldsymbol{q}$ of scalar generalized-coordinate variables. In the case of manipulators containing closed-loops, or when a joint does not admit a global parametrization, not all values of $\boldsymbol{q}$ correspond to feasible configurations, and the configuration space is given by the solution set of a system of non-linear equations

$$
\mathbf{\Phi}(\boldsymbol{q})=\mathbf{0},
$$

which expresses the assembly constraints imposed by the joints [20]. In addition, the possible motion rates of the manipulator, i.e. its feasible instantaneous motions, are characterized by a system of linear equations

$$
L \cdot \boldsymbol{m}=\mathbf{0}
$$

called the velocity equation in [18]. The matrix $\boldsymbol{L}$, which depends on the configuration $\boldsymbol{q}$, can be seen to have the following structure

$$
\boldsymbol{L}=\left[\begin{array}{ccc}
-\boldsymbol{I} & \boldsymbol{A}_{a} & \boldsymbol{A}_{p} \\
\mathbf{0} & \boldsymbol{D}_{a} & \boldsymbol{D}_{p}
\end{array}\right]
$$

The velocity vector $\boldsymbol{m}=\left[\boldsymbol{\Omega}^{o \top}, \boldsymbol{\Omega}^{a \top}, \boldsymbol{\Omega}^{p \top}\right]^{\top}$ has as components $\Omega^{o}$, a vector describing the rate of change of the output motion (for example, the velocity of a point or the angular velocity, or the twist, of an end-effector body), as well as $\boldsymbol{\Omega}^{a}$ and $\boldsymbol{\Omega}^{p}$, describing the rate of change of input and passive motion, respectively (typically, $\boldsymbol{\Omega}^{a}$ and $\boldsymbol{\Omega}^{p}$ are the actuated and unactuated joint speeds, respectively). Such a system of equations can be obtained for any manipulator and therefore can be used for the practical identification of singularities.

In this paper we assume that, for every configuration, the dimension of the output and input velocity vectors are equal to the (global) mobility, $n$, of the kinematic chain (the mobility is equal to the dimension of the configuration space, i.e., to the maximum dimension of its tangent space, wherever such space exists [21]). 
The instantaneous kinematic analysis of a manipulator addresses two main problems:

- The forward instantaneous kinematics problem (FIKP): find $\boldsymbol{m}$ given the input $\boldsymbol{\Omega}^{a}$, and

- The inverse instantaneous kinematics problem (IIKP): find $\boldsymbol{m}$ given the output $\boldsymbol{\Omega}^{\circ}$.

Note that in each case it is required to find all instantaneous parameters of the manipulator, not just the output or input speeds, respectively. Following [18], a configuration is defined as nonsingular when both the FIKP and the IIKP have unique solutions for any input or output. This leads to the identification of three basic types of singularities, namely, input singularities and output singularities, where the FIKP and the IIKP are undetermined, respectively, and configuration-space singularities, where both the FIKP and IIKP become undetermined for any definition of input or output from the given velocity variables. However, depending on the cause of the degeneracy, six substantially different types of singularities can be recognized. These are redundant input (RI), redundant output (RO), impossible input (II), impossible output (IO), increased instantaneous mobility (IIM) and redundant passive motion (RPM) singularities.

Each of the six singularity-type definitions describes an important change in the kinematic properties of the manipulator that occurs in a singular configuration of that type. When the mechanism is in a singularity of type RO or IO (RI or II), the output (input) is indeterminate or restricted. In an IIM-type configuration the instantaneous motion of the manipulator is indeterminate, no matter which $n$ parameters are being controlled. In an RPM-type singularity, the passive motion of the mechanism is indeterminate, which may create problems such as interference with other links and obstacles. It is, therefore, desirable to know whether or not a given configuration belongs to each of these types.

Next section recalls the definition of each type of singularity and defines a system of equations that encodes all singular configurations for each type.

\section{CHARACTERIZATION OF SINGULARITIES}

For the definition of the systems of equations, let $\boldsymbol{L}_{I}, \boldsymbol{L}_{O}$ and $\boldsymbol{L}_{P}$ be the submatrices of $\boldsymbol{L}$ obtained by removing the columns corresponding to the input, output, and both the input and output, respectively.

As proven in $[18,19]$, the singularities of a manipulator can be characterized by those configurations where either the matrix $\boldsymbol{L}_{I}$ or the matrix $\boldsymbol{L}_{O}$ is rank deficient. Note that if a matrix is rank deficient, its kernel has to be non-empty and, in particular, it must include a vector of unit norm. Thus, all singularities can be determined by solving the following two systems of equations:

$$
\left.\left.\begin{array}{c}
\boldsymbol{\Phi}(\boldsymbol{q})=\mathbf{0} \\
\boldsymbol{L}_{I}^{\top} \boldsymbol{\xi}=\mathbf{0} \\
\|\boldsymbol{\xi}\|^{2}=1
\end{array}\right\}, \quad \begin{array}{l}
\boldsymbol{\Phi}(\boldsymbol{q})=\mathbf{0} \\
\boldsymbol{L}_{O}^{\top} \boldsymbol{\xi}=\mathbf{0} \\
\|\boldsymbol{\xi}\|^{2}=1
\end{array}\right\} .
$$

The first equation of each system constraint $\boldsymbol{q}$ to be a feasible configuration of the mechanism, the second and third lines imply the existence of a nonzero vector in the kernel of the corresponding matrix. Note that $\|\boldsymbol{\xi}\|^{2}$ can be any consistent norm, for instance, $\boldsymbol{\xi}^{\top} \boldsymbol{D} \boldsymbol{\xi}$ with $\boldsymbol{D}$ a diagonal matrix where the entries have the proper physical units. There is no need for the norm to be invariant with respect to change of frame or units. In short, the condition $\|\boldsymbol{\xi}\|^{2}=1$ only serves to guarantee that $\boldsymbol{\xi}$ is not $\mathbf{0}$.

The solution of the system on the left in Eq. (3) includes all configurations where the FIKP is undeterminate, corresponding to all input singularities, while the solution of the system on the right includes all output singularities, where the IIKP is undeterminate. Configuration-space singularities will satisfy both systems in (3), as well as any analogous system obtained by deleting $n$ columns in $L$.

The singularity set can be seen to contain six distinct lowlevel singularity types, whose definitions are recalled next. Following each definition, a system of equations encoding the configurations of the corresponding type are derived.

\section{RI-type}

A configuration is a singularity of RI type if there exist a nonzero input, $\boldsymbol{\Omega}^{a} \neq \mathbf{0}$, and a vector $\boldsymbol{\Omega}^{p}$, which satisfy the velocity equation (2) for a zero-output, $\boldsymbol{\Omega}^{o}=\mathbf{0}$, i.e.

$$
\boldsymbol{L}_{O}\left[\begin{array}{l}
\boldsymbol{\Omega}^{a} \\
\boldsymbol{\Omega}^{p}
\end{array}\right]=\mathbf{0} .
$$

This means that the kernel of $\boldsymbol{L}_{O}$ has to include a vector with $\boldsymbol{\Omega}^{a} \neq \mathbf{0}$. Note that, as before, if such a nonzero vector exists, there will also exist a unit vector with nonzero $\Omega^{a}$. Thus $\boldsymbol{q}$ is a singularity of type RI if and only if the system of equations

$$
\left.\begin{array}{l}
\boldsymbol{\Phi}(\boldsymbol{q})=\mathbf{0} \\
\boldsymbol{L}_{O} \boldsymbol{\xi}=\mathbf{0} \\
\|\boldsymbol{\xi}\|^{2}=1
\end{array}\right\}
$$

is satisfied for some value of $\boldsymbol{\xi}=\left[\boldsymbol{\Omega}^{a \top}, \boldsymbol{\Omega}^{p \top}\right]^{\top}$, with $\mathbf{\Omega}^{a} \neq \mathbf{0}$.

The RI singularity is illustrated in Fig. 1 by a four-bar mechanism. In the configuration shown on the left, the output link $D C$ cannot move, since the velocity of point $C$ must be zero, while the instantaneous input, $\omega_{A}$, can have any value.
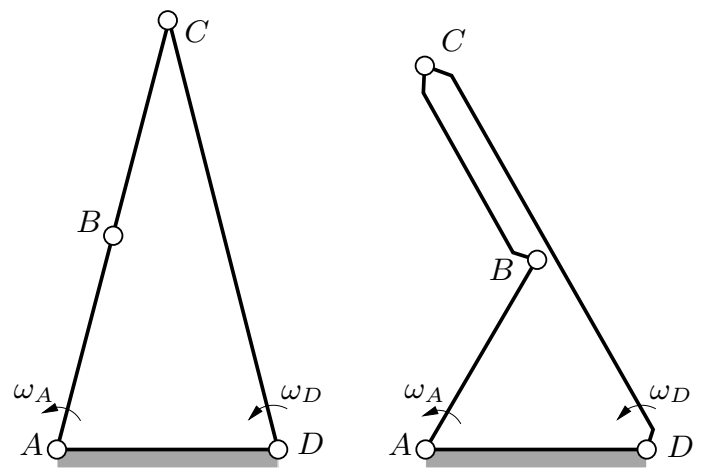

Fig. 1. A four-bar planar mechanism in an RI and IO singularity (left) and in an RO and II singularity (right). $A D=A B=B C=1, C D=2$. 


\section{RO-type}

A configuration is a singularity of RO type if there exist a nonzero output, $\boldsymbol{\Omega}^{o} \neq \mathbf{0}$, and a vector $\boldsymbol{\Omega}^{p}$, which satisfy the velocity equation for a zero-input, $\boldsymbol{\Omega}^{a}=\mathbf{0}$, i.e.

$$
\boldsymbol{L}_{I}\left[\begin{array}{c}
\boldsymbol{\Omega}^{o} \\
\boldsymbol{\Omega}^{p}
\end{array}\right]=\mathbf{0} .
$$

Following a similar reasoning as before, $\boldsymbol{q}$ is of type RO if and only if it satisfies the equations

$$
\left.\begin{array}{c}
\boldsymbol{\Phi}(\boldsymbol{q})=\mathbf{0} \\
\boldsymbol{L}_{I} \boldsymbol{\xi}=\mathbf{0} \\
\|\boldsymbol{\xi}\|^{2}=1
\end{array}\right\}
$$

for some value of $\boldsymbol{\xi}=\left[\boldsymbol{\Omega}^{o \top}, \boldsymbol{\Omega}^{p \top}\right]^{\top}$, with $\boldsymbol{\Omega}^{o} \neq \mathbf{0}$.

The four-bar of Fig. 1 (on the right) is shown in an ROtype singular configuration. The input link $A B$ is locked, while the instantaneous output, $\omega_{D}$, can have any value.

\section{IO-type}

A configuration is a singularity of IO type if there exists a vector $\boldsymbol{\Omega}^{o} \neq \mathbf{0}$ for which the velocity equation cannot be satisfied for any combination of $\boldsymbol{\Omega}^{a}$ and $\boldsymbol{\Omega}^{p}$. In other words, this means that there is a nonzero vector $\left[\boldsymbol{\Omega}^{o \top}, \mathbf{0}^{\top}, \mathbf{0}^{\top}\right]^{\top}$ that cannot be obtained by projection of any vector $\left[\boldsymbol{\Omega}^{o \top}, \boldsymbol{\Omega}^{a \top}, \boldsymbol{\Omega}^{p \top}\right]^{\top}$ belonging to the kernel of $\boldsymbol{L}$.

In order to derive the system of equations for this type, let $\boldsymbol{V}=\left[\boldsymbol{v}_{1}, \ldots, \boldsymbol{v}_{r}\right]$ be a matrix whose columns form a basis of the kernel of $\boldsymbol{L}$. Then, all vectors $\left[\boldsymbol{\Omega}^{o \top}, \mathbf{0}^{\top}, \mathbf{0}^{\top}\right]^{\top}$ that can be obtained by projection of some vector of the kernel of $\boldsymbol{L}$ are in the image space of the linear aplication given by the matrix

$$
\boldsymbol{A}=\left[\begin{array}{ll}
\boldsymbol{I}_{n \times n} & \mathbf{0}
\end{array}\right] \boldsymbol{V},
$$

where $n$ is the dimension of vector $\boldsymbol{\Omega}^{o}$. Thus, a singular configuration is of IO type if the map $\boldsymbol{A}$ is not surjective, i.e. $\boldsymbol{A}$ is rank deficient. In this situation it can be seen that there exists a unit vector $\boldsymbol{\Omega}^{o *}$ in the kernel of $\boldsymbol{A}^{\top}$ and, hence, a vector $\left[\boldsymbol{\Omega}^{o * \top}, \mathbf{0}^{\top}, \mathbf{0}^{\top}\right]^{\top}$ in the kernel of $\boldsymbol{V}^{\top}$. Such a vector is orthogonal to all vectors $\boldsymbol{v}_{1}, \ldots, \boldsymbol{v}_{r}$, so it must belong to the image of $\boldsymbol{L}^{\top}$. In conclusion, there must exist a nonzero vector $\boldsymbol{\Omega}^{o *}$ satisfying

$$
\boldsymbol{L}^{\top} \boldsymbol{\psi}=\left[\begin{array}{c}
\boldsymbol{\Omega}^{o *} \\
\mathbf{0} \\
\mathbf{0}
\end{array}\right],
$$

for some vector $\boldsymbol{\psi}$. By letting $\boldsymbol{\psi}=\left[\boldsymbol{\psi}_{1}^{\top}, \boldsymbol{\psi}_{2}^{\top}\right]^{\top}$, where $\boldsymbol{\psi}_{1}$ has the same dimension as $\Omega^{o *}$, and by taking advantage of the structure of $\boldsymbol{L}$, it can be seen that $\boldsymbol{\psi}_{1}=-\boldsymbol{\Omega}^{o *}$. Therefore a configuration, $\boldsymbol{q}$, is an IO-type sigularity if and only if it satisfies

$$
\left.\begin{array}{l}
\boldsymbol{\Phi}(\boldsymbol{q})=\mathbf{0} \\
\boldsymbol{L}_{O}^{\top} \boldsymbol{\xi}=\mathbf{0} \\
\|\boldsymbol{\xi}\|^{2}=1
\end{array}\right\}
$$

for some $\boldsymbol{\xi}=\left[-\boldsymbol{\Omega}^{o * \top}, \boldsymbol{\psi}_{2}^{\top}\right]^{\top}$, with $\boldsymbol{\Omega}^{o *} \neq \mathbf{0}$. For all solutions of this system, the obtained value of $\boldsymbol{\Omega}^{o *}$ will be a non-feasible output of the manipulator at the corresponding configuration.

A singular configuration of type IO can be seen by considering again the four-bar configuration shown in the left-hand-side part of Fig. 1. The only feasible output is zero, and any non-zero output is impossible.

\section{II-type}

A configuration is a singularity of type II if there exists a vector $\boldsymbol{\Omega}^{a} \neq \mathbf{0}$ for which the velocity equation cannot be satisfied for any combination of $\boldsymbol{\Omega}^{o}$ and $\boldsymbol{\Omega}^{p}$. Following similar observations as for the IO type, a configuration $\boldsymbol{q}$ is a singularity of II type if and only if there exists a nonzero vector $\Omega^{a *}$ such that

$$
\boldsymbol{L}^{\top} \boldsymbol{\psi}=\left[\begin{array}{c}
\mathbf{0} \\
\boldsymbol{\Omega}^{a *} \\
\mathbf{0}
\end{array}\right],
$$

for some $\psi$. By taking advantage of the structure of $\boldsymbol{L}$ as before, it can be seen that $\psi_{1}=\mathbf{0}$, and the previous condition can be written as

$$
\left[\begin{array}{l}
\boldsymbol{D}_{a}^{\top} \\
\boldsymbol{D}_{p}^{\top}
\end{array}\right] \boldsymbol{\psi}_{2}=\left[\begin{array}{c}
\boldsymbol{\Omega}^{a *} \\
\mathbf{0}
\end{array}\right]
$$

In principle, there will be many combinations of $\boldsymbol{\psi}_{2}$ and $\boldsymbol{\Omega}^{a *}$ satisfying this condition, but since $\psi_{2}$ must be nonzero and in the kernel of $\boldsymbol{D}_{p}^{\top}$, it can be chosen of unit norm. Hence, II-type singularities are exactly the configurations that satisfy

$$
\left.\begin{array}{c}
\mathbf{\Phi}(\boldsymbol{q})=\mathbf{0} \\
{\left[\begin{array}{l}
\boldsymbol{D}_{a}^{\top} \\
\boldsymbol{D}_{p}^{\top}
\end{array}\right] \boldsymbol{\psi}_{2}=\left[\begin{array}{c}
\boldsymbol{\Omega}^{a *} \\
\mathbf{0}
\end{array}\right]} \\
\left\|\boldsymbol{\psi}_{2}\right\|^{2}=1
\end{array}\right\},
$$

for some $\boldsymbol{\psi}_{2}$ with $\boldsymbol{\Omega}^{a *} \neq \mathbf{0}$.

The four-bar of Fig. 1 (right) is shown in an II-type singular configuration: the input link is locked, and any nonzero input is impossible.

\section{RPM-type}

A configuration is a singularity of type RPM if there exists a nonzero $\Omega^{p}$ vector which satisfies the velocity equation for a zero input and a zero output, i.e. $\boldsymbol{L}_{P} \boldsymbol{\Omega}^{p}=\mathbf{0}$. This will happen when the kernel of $\boldsymbol{L}_{P}$ is non-zero and, thus, the following system of equations

$$
\left.\begin{array}{c}
\boldsymbol{\Phi}(\boldsymbol{q})=\mathbf{0} \\
\boldsymbol{L}_{P} \boldsymbol{\Omega}^{p}=\mathbf{0} \\
\left\|\boldsymbol{\Omega}^{p}\right\|^{2}=1
\end{array}\right\}
$$

encodes all RPM-type singularities $\boldsymbol{q}$.

Fig. 2 shows a 1-dof mechanism with three sliders. The velocity of point $A$ is the input, the velocity of $B$ is the output, and the velocity of $C$ is a passive joint rate. In the configuration shown on the right, both points $A$ and $B$ must have zero velocity, while the velocity of point $\mathrm{C}$ can be nonzero. Hence, motion of the manipulator is possible when both the input and the output are zero, and the manipulator is in an RPM-type singularity. 

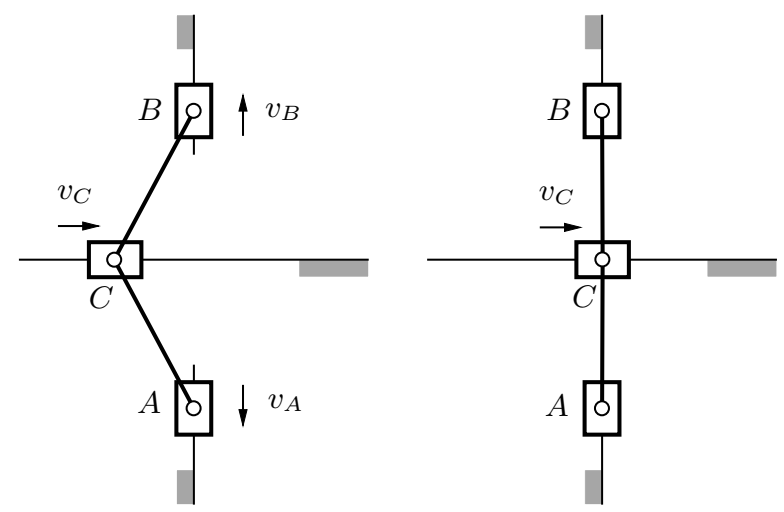

Fig. 2. A 1-dof planar manipulator in a non-singular configuration (left) and in an RPM singular configuration (right).

\section{IIM-type}

A configuration is a singularity of type IIM if $\boldsymbol{L}$ is rank deficient. In fact, it corresponds to configurations where the instantaneous mobility is greater than the number of degrees of freedom. The definition directly allows to write the system of equations

$$
\left.\begin{array}{r}
\boldsymbol{\Phi}(\boldsymbol{q})=\mathbf{0} \\
\boldsymbol{L}^{\top} \boldsymbol{\xi}=\mathbf{0} \\
\|\boldsymbol{\xi}\|^{2}=1
\end{array}\right\}
$$

that will be satisfied for some $\boldsymbol{\xi}$ by a configuration $\boldsymbol{q}$ if and only if it is an IIM-type singularity.

A four-bar in the flattened configuration of Fig. 3 obtains a transitory mobility of 2 and, thus, it is in an IIM-type singularity.

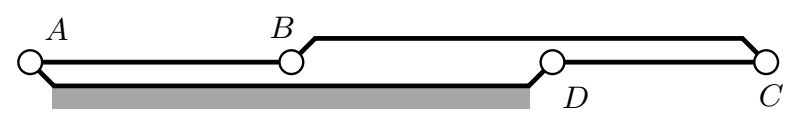

Fig. 3. An IIM-type singularity.

\section{ISOLATION OF SINGULARITIES}

A numerical method able to solve the previous systems of equations is next described. The method consists in first formulating the system of equations that characterizes each singularity type in an appropriate way, allowing to compute an initial box bounding the location of all solutions, and then using a numerical technique that exploits the structure of the system to isolate the set of singularities at the desired resolution.

\section{A. Equation formulation}

All systems in (3)-(9) can be formulated so they adopt the form of a polynomial system of quadratic equations (i.e. equations where only monomials of the form $a, a^{2}$, or $a b$ appear, where $a$ and $b$ refer to any two of the variables). In addition, it will be seen that each variable in the system can only take values within a limited interval, so that from the Cartesian product of all such intervals one can define a box $\mathcal{B}$ that initially bounds all solutions of the system. This allows the use of a particularly simple technique to compute the solution of the systems numerically.

First note that the structure of all systems (3)-(9) is very similar. The first line is always Eq. (1), because all solution points must correspond to feasible configurations of the manipulator. The second line always involves matrix $\boldsymbol{L}$, or one of its sub-matrices, and the third line corresponds to the norm of some vector. For a manipulator involving lower pairs of any kind, except the helical pair, the formulation proposed in [22] makes Eq. (1) directly adopt the form of a polynomial system of quadratic equations. The second line will be quadratic too, because all the entries in $\boldsymbol{L}$ are linear terms, and the third line is directly a quadratic expression.

Moreover, as proven in [22], one can always define a possible interval of values for all variables $\boldsymbol{q}$ used in such a formulation. Note, additionally, that the entries of the vector in the last line of each system are also bounded in the range $[-1,1]$. In the case of system (7), the feasibility intervals for the entries of $\Omega^{a}$ can be readily obtained by mapping the known intervals using $D_{a}^{\top} \psi_{2}=\Omega^{a}$.

Thus, in order to apply the numerical method, it only remains to model the fact that in systems (4)-(7), $\Omega^{a}$ or $\Omega^{o}$ must be different from zero. Since the technique can also handle non-strict inequalities, this can be done by setting $\left\|\boldsymbol{\Omega}^{a}\right\|^{2} \geq \epsilon$ for systems (4) and (7), and $\left\|\boldsymbol{\Omega}^{o}\right\|^{2} \geq \epsilon$ for systems (5) and (6), where $\epsilon$ is a sufficiently small value. By using this inequality some singularities might be lost, but $\epsilon$ can be made arbitrarily small, reducing this set of missed solutions to a negligible size.

Finally, for ease of explanation, note that all systems (3)(9) can be written as

$$
\boldsymbol{F}(\boldsymbol{x})=\mathbf{0},
$$

where, in each case, $\boldsymbol{x}$ contains all the variables involved in the system. Let us now define the changes of variables $p_{i}=x_{i}^{2}$ for each quadratic monomial and $b_{k}=x_{i} x_{j}$ for each bilinear monomial in (10), in order to allow transforming the system into the expanded form

$$
\left.\begin{array}{l}
\boldsymbol{\Lambda}(\boldsymbol{x})=0 \\
\boldsymbol{\Gamma}(\boldsymbol{x})=0
\end{array}\right\}
$$

where $\boldsymbol{\Lambda}(\boldsymbol{x})=0$ is a collection of linear equations in $\boldsymbol{x}$ and $\boldsymbol{\Gamma}(\boldsymbol{x})=0$ is a collection of quadratic equations, each of which adopts one of the two forms $x_{k}=x_{i}^{2}$ or $x_{k}=x_{i} x_{j}$.

\section{B. Numerical solution}

The algorithm for solving system (11) recursively applies two operations on the computed box $\mathcal{B}$ : box shrinking and box splitting. Using box shrinking, portions of $\mathcal{B}$ containing no solution are eliminated by narrowing some of its defining intervals. This process is repeated until either the box is reduced to an empty set, in which case it contains no solution, or the box is "sufficiently" small, in which case it is considered a solution box, or the box cannot be "significantly" reduced, in which case it is bisected into two sub-boxes via box splitting (which simply bisects its largest interval). To converge to all solutions, the whole process is 
(a)

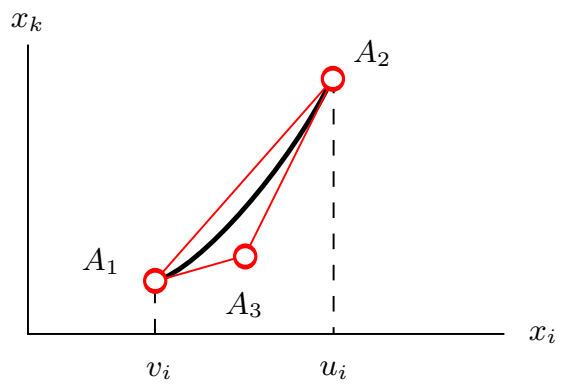

(b)

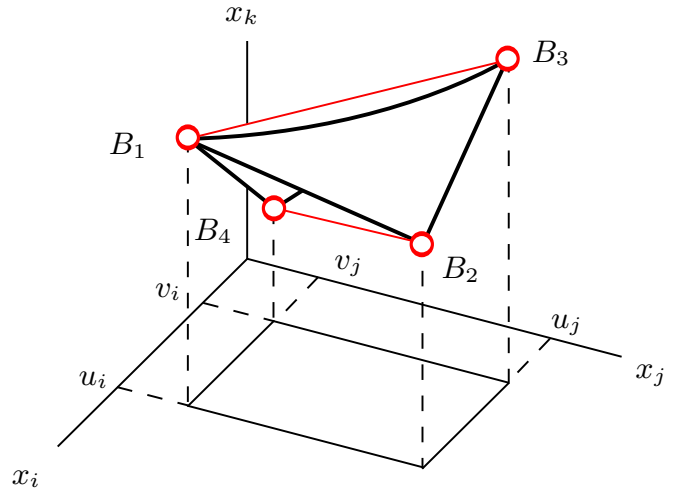

Fig. 4. Polytope bounds within box $\mathcal{B}_{c}$.

recursively applied to the new sub-boxes, until one obtains a collection of solution boxes whose side lengths are below a given threshold $\sigma$.

The crucial operation in this scheme is box shrinking, which is implemented as follows. Note first that the solutions falling in some sub-box $\mathcal{B}_{c} \subseteq \mathcal{B}$ must lie in the linear variety defined by $\boldsymbol{\Lambda}(\boldsymbol{x})=0$. Thus, we may shrink $\mathcal{B}_{c}$ to the smallest possible box bounding this variety inside $\mathcal{B}_{c}$. The limits of the shrunk box along, say, dimension $x_{i}$ can be found by solving the two linear programs

LP1: Minimize $x_{i}$, subject to: $\boldsymbol{\Lambda}(\boldsymbol{x})=0, \boldsymbol{x} \in \mathcal{B}_{c}$

LP2: Maximize $x_{i}$, subject to: $\boldsymbol{\Lambda}(\boldsymbol{x})=0, \boldsymbol{x} \in \mathcal{B}_{c}$.

However, observe that $\mathcal{B}_{c}$ can be further reduced, because the solutions must also satisfy all equations $x_{k}=x_{i}^{2}$ and $x_{k}=x_{i} x_{j}$ in $\boldsymbol{\Gamma}(\boldsymbol{x})=0$. These equations can be taken into account by noting that, if $\left[v_{i}, u_{i}\right]$ denotes the interval of $\mathcal{B}_{c}$ along dimension $x_{i}$, then:

1) The portion of the parabola $x_{k}=x_{i}^{2}$ lying inside $\mathcal{B}_{c}$ is bound by the triangle $A_{1} A_{2} A_{3}$, where $A_{1}$ and $A_{2}$ are the points where the parabola intercepts the lines $x_{i}=v_{i}$ and $x_{i}=u_{i}$, and $A_{3}$ is the point where the tangent lines at $A_{1}$ and $A_{2}$ meet (Fig. 4a).

2) The portion of the hyperbolic paraboloid $x_{k}=x_{i} x_{j}$ lying inside $\mathcal{B}_{c}$ is bound by the tetrahedron $B_{1} B_{2} B_{3} B_{4}$, where the points $B_{1}, \ldots, B_{4}$ are obtained by lifting the corners of the rectangle $\left[v_{i}, u_{i}\right] \times\left[v_{j}, u_{j}\right]$ vertically to the paraboloid (Fig. 4b).

Thus, linear inequalities corresponding to these bounds can be added to LP1 and LP2, which usually produces a much larger reduction of $\mathcal{B}_{c}$ or, if one of the linear programs is found unfeasible, its complete elimination. In this step, the inequalities needed in systems (4)-(7) can also be taken into account.

The collection $B$ of all solution boxes, which is returned as output upon termination, is said to form a box approximation of the solution set of Eq. (11), because the boxes in $B$ form a discrete envelop of a such set, whose accuracy can be adjusted through the $\sigma$ parameter. Notice that the algorithm is complete, in the sense that it will succeed in isolating all solution points of Eq. (11) accurately, provided that a smallenough value for $\sigma$ is used.

\section{AN ILLUSTRATIVE EXAMPLE}

The 2-dof planar manipulator shown in Fig. 5 is used here to illustrate the computation of each one of the singularity types. The inputs are the joint velocities at $A$ and $E$, and the output is the motion of point $G$. The loop equations $\boldsymbol{\Phi}(\boldsymbol{q})=0$ are written as

$$
\begin{aligned}
& 0=-x+2 \cos \theta_{D}+\frac{3}{2} \cos \theta_{C} \\
& 0=-y+2 \sin \theta_{D}+\frac{3}{2} \sin \theta_{C} \\
& 0=\cos \theta_{A}+\cos \theta_{B}-2 \cos \theta_{D}-1, \\
& 0=\sin \theta_{A}+\sin \theta_{B}-2 \sin \theta_{D} \\
& 0=2 \cos \theta_{D}+\frac{3}{2} \cos \theta_{C}+2 \cos \theta_{G}-3 \cos \theta_{E}-1, \\
& 0=2 \sin \theta_{D}+\frac{3}{2} \sin \theta_{C}+2 \sin \theta_{G}-3 \sin \theta_{E},
\end{aligned}
$$

where $\theta_{A}, \theta_{B}, \theta_{C}, \theta_{D}, \theta_{E}$ and $\theta_{G}$ are the counterclockwise angles of links $A B, B C, C G, D C, E F$, and $G F$, respectively, relative to the ground, and $x, y$ are the coordinates of point $G$ relative to a fixed frame centered in $D$. The velocity equation of the manipulator may be obtained, for instance, by differentiating (12) with respect to all variables, but it could also be obtained using the twist loop equations, or by any other means. In order to achieve the desired quadratic formulation, the changes of variables $c_{\tau}=\cos \theta_{\tau}$ and $s_{\tau}=$ $\sin \theta_{\tau}$ can be applied for all $\tau \in\{A, B, C, D, E, G\}$. Since the variables $c_{\tau}$ and $s_{\tau}$ represent the cosine and sine of a variable, the circle equations $c_{\tau}^{2}+s_{\tau}^{2}=1$ are introduced in

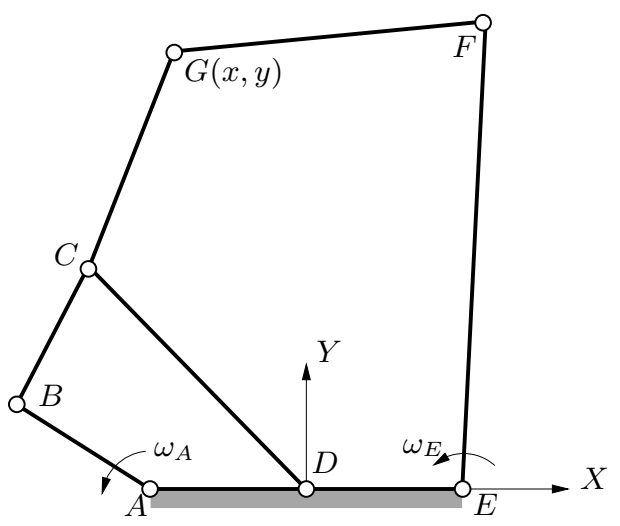

Fig. 5. A 2-dof planar manipulator. The link dimensions are $A B=A D=$ $B C=D E=1, C D=F G=2, C G=1.5$ and $E F=3$. 


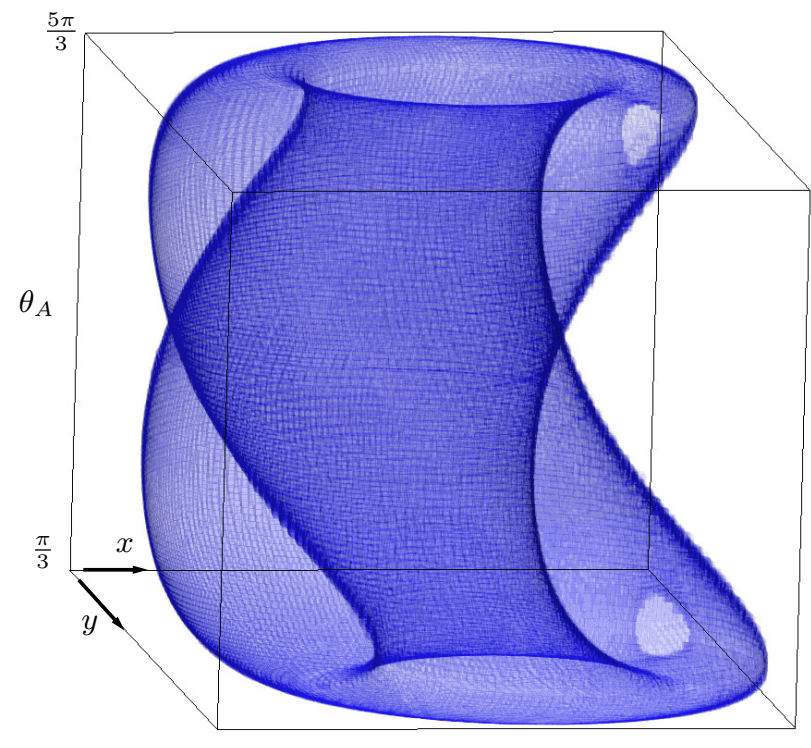

Fig. 6. Two-dimensional configuration space of the manipulator computed at $\sigma=0.1$. Two holes can be seen, whose boundary corresponds to configurations where $E, F$ and $G$ are aligned.

the systems encoding each one of the singularity types, for all angles.

Given that the manipulator has two degrees of freedom, its configuration space is a surface, shown in Fig. 6 projected onto the $x, y$ and $\theta_{A}$ variables. This surface was obtained from the computation of all solutions of (1) using the same numerical technique presented in the previous section. Note that by fixing $x, y$, and $\theta_{A}$, there are still two possible positions of point $F$, so that most of the points in this projection correspond, in fact, to two different configurations of the manipulator. Only the points where $E, F$, and $G$ are aligned represent a single configuration, and these are exactly the boundaries of the two "holes" that the surface presents.

The singularity set is generally of lower dimension than the configuration space, so that only curves or points are to be expected in the solution set of all systems of equations. The result of the computation of each type of singularity is shown in Figs. 7 and 8, projected onto the output and one input $\left(x, y, \theta_{A}\right)$, and onto the output only, respectively. In Fig. 7, the configuration space is shown in blue, separated in two parts so that a cross-section can be seen, but both parts are actually connected through $\pi$ and $-\pi$ as in Fig. 6. The grey area in Fig. 8 represents all attainable positions of point $G$, i.e. the workspace of the manipulator.

As it turns out, this manipulator contains no IIM configurations, and the computation of this type of singularity gives no box as output. On the contrary, there are eight distinct RPM singularities, which in these projections appear coincident in pairs as four orange boxes, corresponding to the two possible locations of $F$. Using a different projection, for instance onto $\left(\theta_{A}, \theta_{D}, \theta_{E}\right)$, the eight boxes appear separated.

The green curves correspond to singularities that are both of type RI and of type IO. These configurations can be seen to contour the two "holes" of the configuration space in this projection. The red curves correspond to configurations simultaneously belonging to the RO and the II type. Even if the curves for RI and IO seem to coincide everywhere, there are some IO configurations that are not of RI type, and the same happens for II and RO singularities, respectively. This is illustrated in Fig. 7 with a magnifying bubble that shows only the output of computing RI singularities. These gaps on the curves of RI and RO, which can be found by properly adjusting the $\epsilon$ parameter, coincide with the location of the RPM singularities and, hence, the RPM singularities are also of type II and IO (but not of type RI or RO). Fig. 8a shows an example of an (RPM, II, IO) singularity, while Fig. 8b and Fig. 8c show examples of (RI, IO) and (RO, II) singularities, respectively.

Fig. 7 also shows yellow curves that correspond to configurations where points $D, B$ and $G$ are aligned. In these configurations there is only one possible location of point $C$. Hence, these configurations, together with those where $E, F$, and $G$ are aligned, allow the transition between different assembly modes. In the figure, these configurations coincide with the points of self-intersection of the projection of the configuration space on the $\left(x, y, \theta_{A}\right)$ space. The configuration space itself has no self-intersections as there are no configuration-space, or IIM-type, singularities. The yellow points are only singularities of the projection map.

Using the same code of colors, Figs. 9 and 10 show the projection of the results onto the the 3-dimensional space of the two input angles and one passive joint angle $\left(\theta_{A}, \theta_{E}\right.$, $\left.\theta_{D}\right)$ and onto the 2-dimensional input space only. The eight RPM singularities appear separated. As before, for each $\theta_{A}$, $\theta_{E}$, and $\theta_{D}$ fixed, there are still two possible locations of point $C$ in general, and almost all points in this projection correspond to two distinct configurations of the manipulator. It can be seen that the configuration space presents four "holes" in these projections. These four contours are made of those configurations where $G, C$, and $F$ are aligned and there is only one possibility for $C$. Note that none of these "holes" coincides with one in the previous projection, but, once again, crossing each curve allows the transition between two different assembly modes. One can imagine the two assembly modes as the two "sides" of the surface of the configuration-space projection. To "get to the opposite side", i.e., to change assembly mode, the motion curve must "go through a hole".

\section{CONCLusions}

This paper has introduced a method for the numerical computation and detailed classification of the entire singularity set of a mechanism with arbitrary geometry and nonhelical lower-pair joints. Singularity subsets corresponding to the six singularity types are computed. The approach, based on the segmentation of the configuration space, is particularly practical and useful for lower-dof mechanisms. In higher-dimensional problems, as is the case with any method, computation times increase and visualization becomes difficult. To circumvent this, it is possible to consider lower-dimensional sections of the configuration space. 


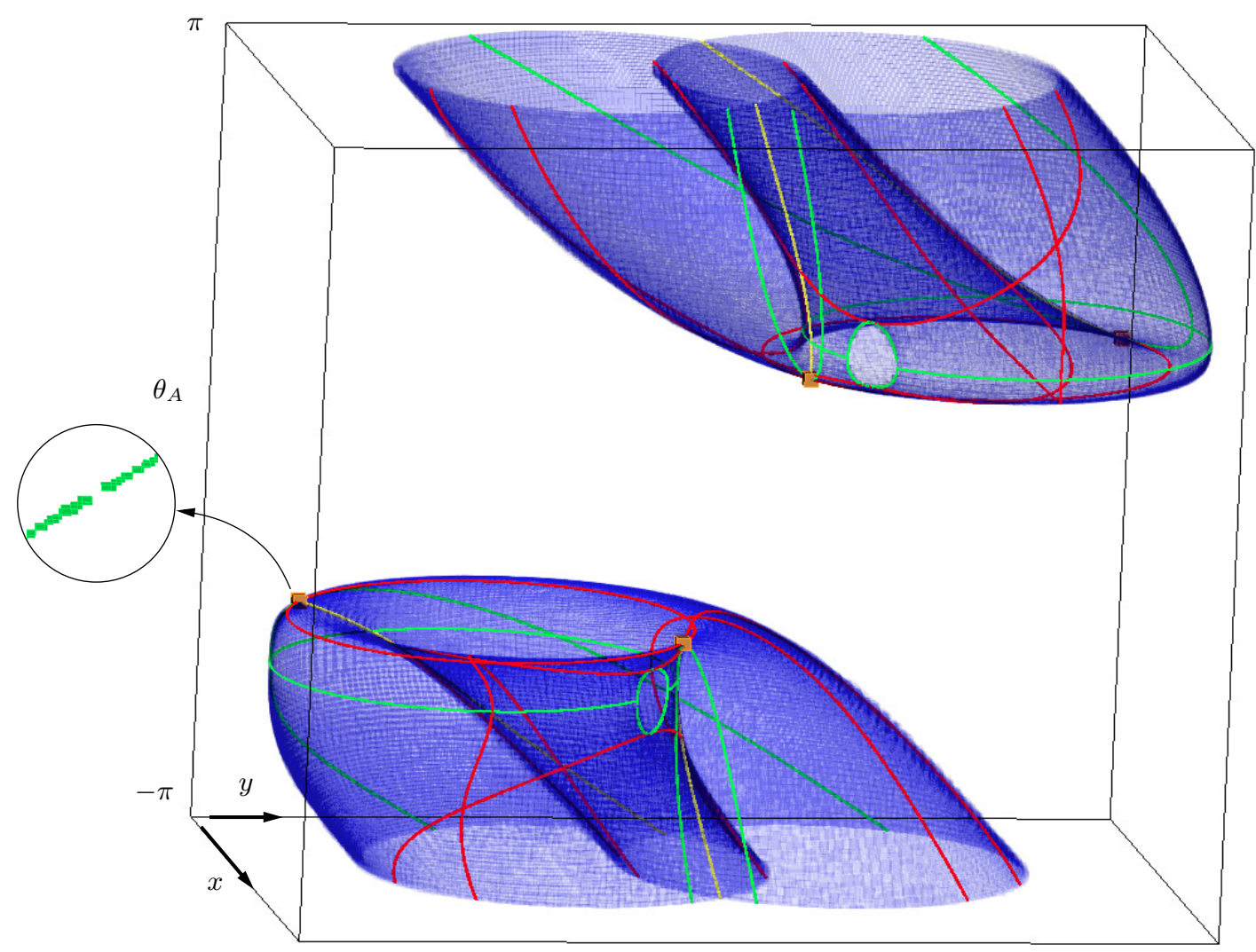

Fig. 7. All singular configurations are shown on the configuration space of the manipulator. Green: (RI,IO), red: (RO, II), orange: (RPM, II, IO). There are no IIM-type singularities.

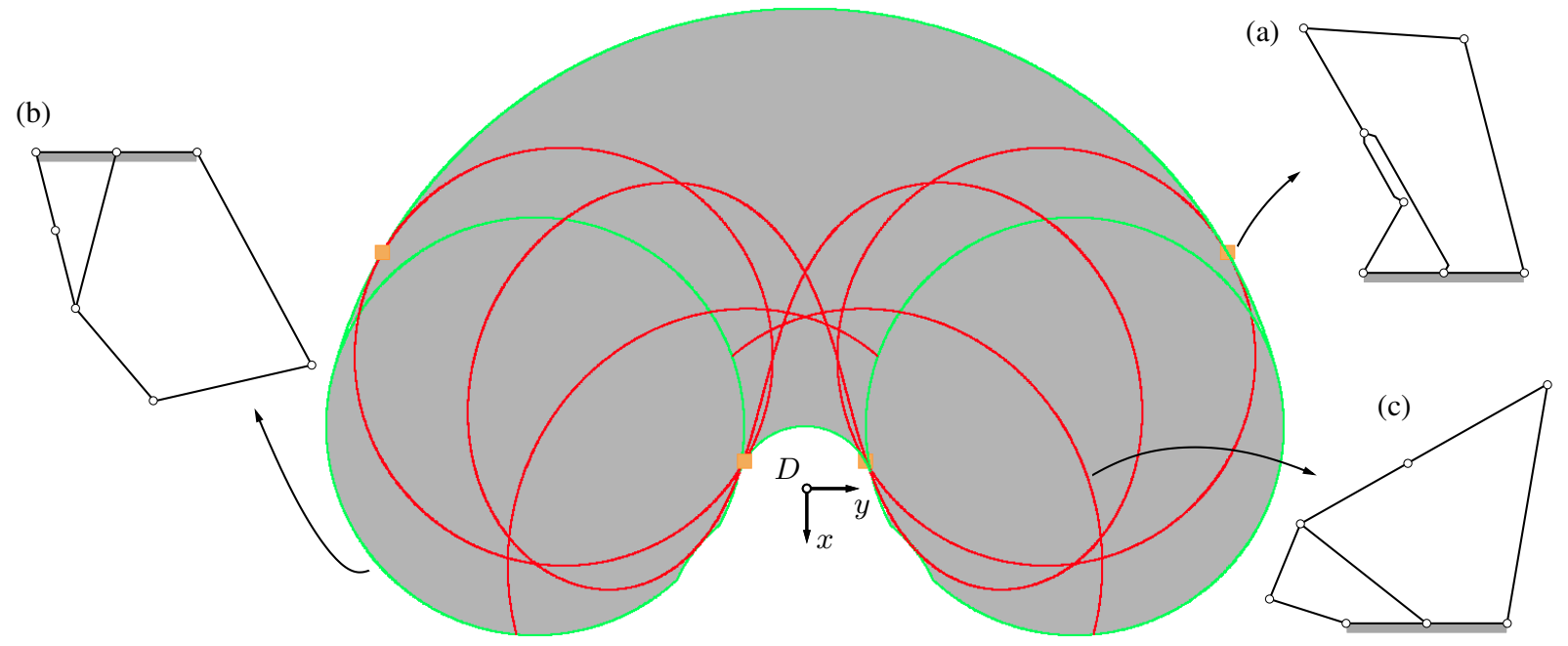

Fig. 8. Projection to the $(x, y)$ space. (a) RPM-IO-II; (b) RI-IO; (c) RO-II

\section{REFERENCES}

[1] C. Gosselin, "Determination of the workspace of 6-DOF parallel manipulators," ASME Journal of Mechanical Design, vol. 112, p. 331, 1990.

[2] J. P. Merlet, "Geometrical determination of the workspace of a constrained parallel manipulator," in Advances in Robot Kinematics, 1992, pp. 326-329.

[3] J. P. Merlet, C. M. Gosselin, and N. Mouly, "Workspaces of planar parallel manipulators," Mechanism and Machine Theory, vol. 33, no. 1-2, pp. 7-20, 1998.
[4] J.-P. Merlet, "Determination of 6D workspaces of Gough-type parallel manipulator and comparison between different geometries," International Journal of Robotics Research, vol. 18, no. 9, pp. 902-916, 1999.

[5] I. Bonev and J. Ryu, "A new approach to orientation workspace analysis of 6-DOF parallel manipulators," Mechanism and Machine Theory, vol. 36, no. 1, pp. 15-28, 2001.

[6] J. A. Snyman, L. J. du Plessis, and J. Duffy, "An optimization approach to the determination of the boundaries of manipulator workspaces," ASME Journal of Mechanical Design, vol. 122, no. 4, pp. 447-456, 2000.

[7] I. A. Bonev and C. M. Gosselin, "Analytical determination of the 


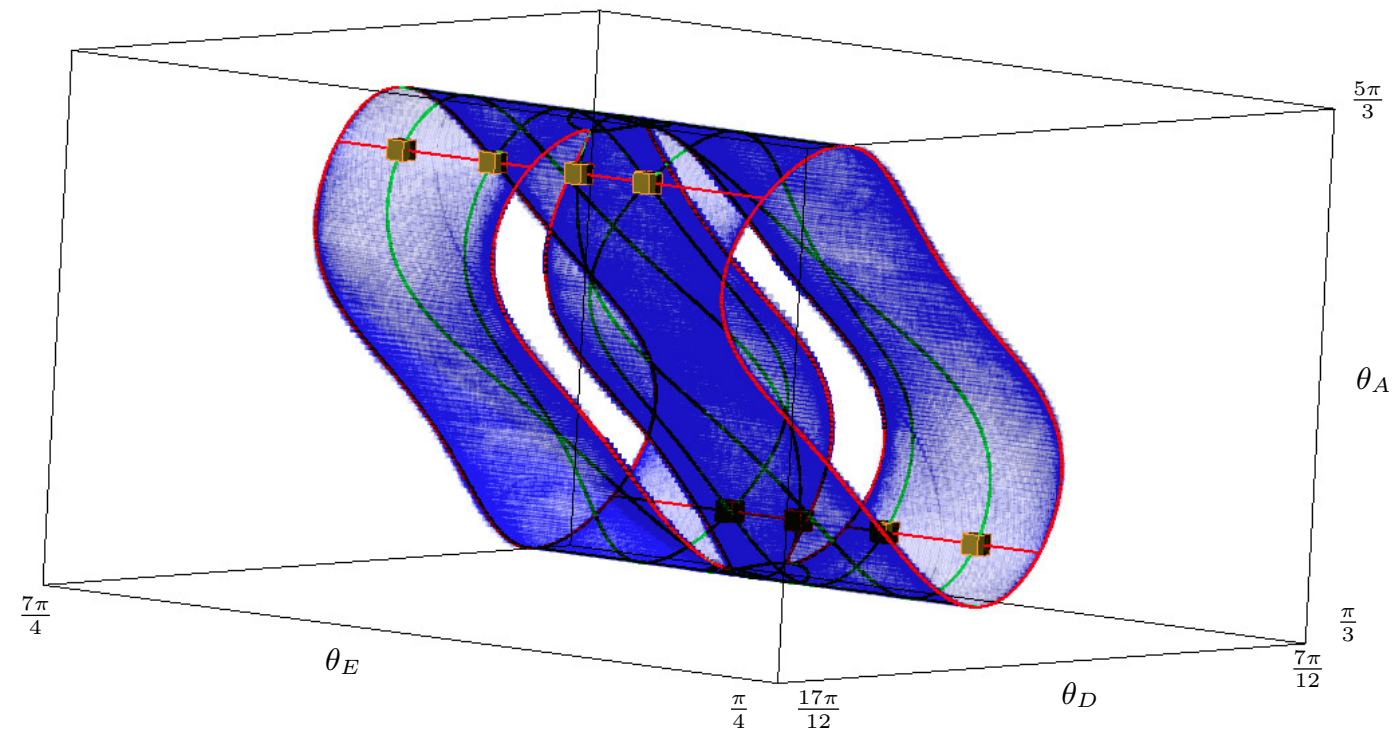

Fig. 9. Projection to the $\left(\theta_{A}, \theta_{E}, \theta_{D}\right)$ space. Green: RI and IO, red: RO and II, orange: RPM. There are no IIM singularities.

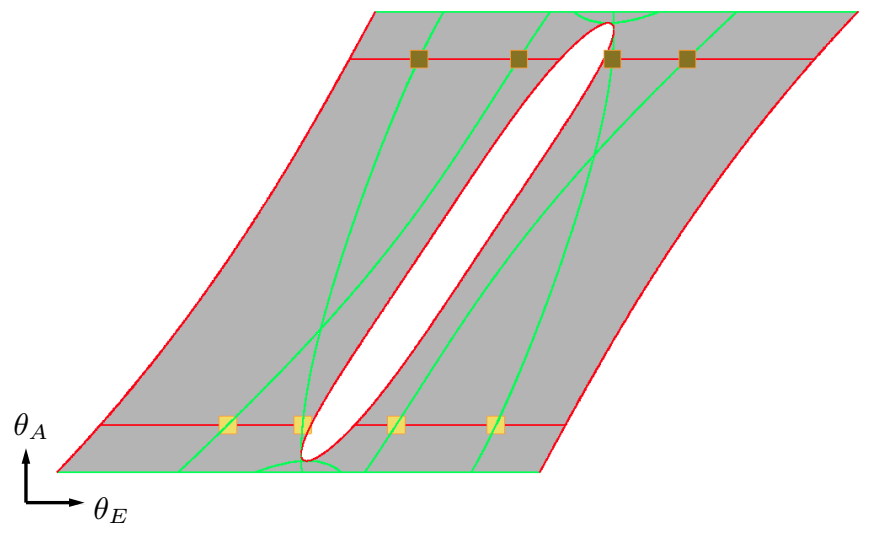

Fig. 10. Projection to the $\left(\theta_{A}, \theta_{E}\right)$ space.

workspace of symmetrical spherical parallel mechanisms," IEEE Transactions on Robotics, vol. 22, no. 5, pp. 1011-1017, 2006.

[8] M. Zein, P. Wenger, and D. Chablat, "An exhaustive study of the workspace topologies of all $3 \mathrm{R}$ orthogonal manipulators with geometric simplifications," Mechanism and Machine Theory, vol. 41, no. 8, pp. 971-986, 2006.

[9] E. Ottaviano, M. Husty, and M. Ceccarelli, "Identification of the workspace boundary of a general 3-R manipulator," ASME Journal of Mechanical Design, vol. 128, no. 1, pp. 236-242, 2006.

[10] E. J. Haug, C.-M. Luh, F. A. Adkins, and J.-Y. Wang, "Numerical algorithms for mapping boundaries of manipulator workspaces," ASME Journal of Mechanical Design, vol. 118, pp. 228-234, 1996.
[11] H. Li, C. Gosselin, M. Richard, and B. St-Onge, "Analytic form of the six-dimensional singularity locus of the general Gough-Stewart platform," ASME Journal of Mechanical Design, vol. 128, pp. 279288, 2006.

[12] J.-P. Merlet, "A formal-numerical approach for robust in-workspace singularity detection," IEEE Transactions on Robotics, vol. 23, no. 3, pp. 393-402, 2007.

[13] Q. Jiang and C. Gosselin, "Determination of the maximal singularityfree orientation workspace for the Gough-Stewart platform," Mechanism and Machine Theory, vol. 44, no. 6, pp. 1281-1293, 2009.

[14] C. Gosselin and J. Angeles, "Singularity analysis of closed-loop kinematic chains," IEEE Transactions on Robotics and Automation, vol. 6, pp. 281-290, 1990.

[15] F. Park and J. Kim, "Manipulability of closed kinematic chains," ASME Journal of Mechanical Design, vol. 120, pp. 542-548, 1996.

[16] _ - "Singularity analysis of closed kinematic chains," ASME Journal of Mechanical Design, vol. 121, pp. 32-38, 1999.

[17] D. Zlatanov, R. Fenton, and B. Benhabib, "A unifying framework for classification and interpretation of mechanism singularities," ASME Journal of Mechanical Design, vol. 117, pp. 566-572, 1995.

[18] D. Zlatanov, "Generalized singularity analysis of mechanisms," Ph.D. dissertation, University of Toronto, 1998.

[19] D. Zlatanov, B. Benhabib, and R. Fenton, "Identification and classification of the singular configurations of mechanisms," Mechanism and Machine Theory, vol. 33, pp. 743-760, 1998.

[20] J. G. De Jalón and E. Bayo, Kinematic and Dynamic Simulation of Multibody Systems. Springer Verlag, 1993.

[21] J. Selig, Geometric fundamentals of robotics. Springer, 2005.

[22] J. M. Porta, L. Ros, and F. Thomas, "A linear relaxation technique for the position analysis of multi-loop linkages," IEEE Transactions on Robotics, vol. 25, no. 2, pp. 225-239, 2009. 